\title{
MULTIUSER ARRAY BEAMFORMING BASED ON A NEURAL NETWORK MAPPING*
}

\author{
Ana Pérez-Neira, Miguel A. Lagunas. \\ Department of Signal Theory and Communications, Universitat Politecnica de Catalunya \\ 08071 BARCELONA, SPAIN.e-mail: anuska@tsc.upc.es
}

\begin{abstract}
This paper addresses the problem of simultaneous multiuser service. To find a feasable solution to the collision problem, two Time Reference Multibeamformer systems are developed (BPSK TRMB and Frequency framing TRMB). Based on a two-stage architecture, they enlarge the potential of existing separating neural networks and the algorithm (EM). Their goal is to perform a low computational simultaneous digital demodulation of users. The results support the frequency framing $T R M B$ as the more robust system, which is able to give service to users in very degradated SNR scenarios; only the decoupling weights and their corresponding High Order Statistics learning is the overhead from a pure superposition of single channel receivers.
\end{abstract}

\section{INTRODUCTION}

The source separation problem, reported some years ago by Y.Bar-Ness [1] and C.Jutten/J.Herault [2], has been evolved as a major tool in signal processing. Focussing cross-coupled polarisation and signal separation in a neural network application respectively, the so-called feed-forward (FF) and feed-backward (FB) structures achieve in a recursive computational way an effective blind separation of independent sources or signals from a given physical scenario.

In this paper we further support our believe which is just to define the above structures as a direct implementation of the estimate and maximise algorithm (EM) [3]. Yet preserving the basic framework of the source separation we enlarge the potential of the EM algorithm by a two cooperative stage structure (with respect to the single one of Bar-Ness or Jutten) following the neural network mapping theorem due to A.Kolmogorov [4].

The form of this general two-stage architecture depends on the application. In [5] the authors developed a design for simultaneous DOA estimation based on the mentioned architecture. Now, in this work, the problem of narrow-band Time Reference Multibeamformer (TRMB) is addressed enlarging the techniques developed in [6]

*Supported by National Research Plan of Spain,CICYT, Grant number TIC92-0800-C05-05

\section{TIME REFERENCE MULTIBEAMFORMING}

The basic TRB scheme is shown in figure (1). The goal is to form a dedicated beam for the user which is asking for a communication link. For this purpose, the user issues a reference signal, $s_{i}$, to the beamformer in order to allow the receiver to locally regenerate it with a demodulator-modulator chain. Once the desired signal is ready, $r_{i}$, it will be compared with the array output $\left(w^{H} . x\right)$ in order to garantee an error or residual signal. This residual drives an adaptive algorithm to steer the desired source and null the

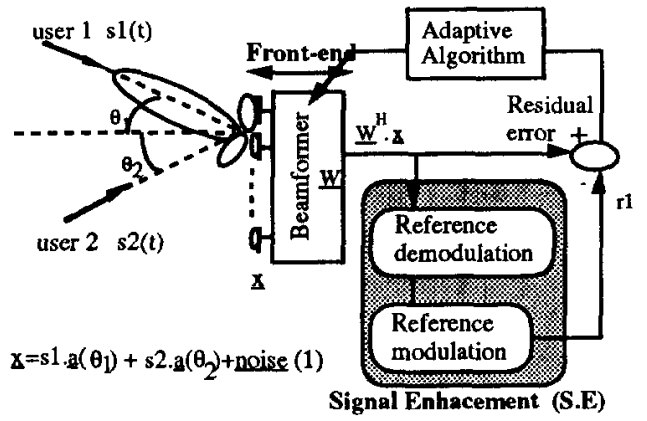

Figure 1.Single user Time Reference Beamformer (TRB)

interferences as much as possible. In this way the SNIR (Signal to Noise and Interference Ratio) requirements in the communication link decrease due to the selective directivity introduced by the beam. Additionally, the efficiency of the link would be increased if multiple TRBs are set to work parallely (TRMB). However some problems arise due to the collision among simultaneous users when asking for connection. To get this problems round, the authors put forward to insert a signal separation network between the front end and the reference enhancer part. Hence, the final architecture will consist of two stages: a first separating stage based on spatial descrimination and statistical independence properties and a second enhancing or optimization stage which will clean the separated references from the noise.

In this work, both feed-forward (FF) and feedbackward (FB) structures will be considered for the 
separating network, which are only applicable for separation of signals and not for noise reduction. These networks differ mainly in their processing bandwith and convergence time [1]: although slower, the FF network involves no loops and, therefore, avoids the inherent delay of the FB solution. Both structures will be used in this work for BPSK Time Reference Beamformer or for frequency-framed TRB respectively. Their performance will depend on how well the architecture is able to solve the noise reduction problem.

\section{3,BPSK TIME REFERENCE BEAMFORMING}

If in a TRB system, the reference signal is inherent to the information message that the user wants to transmit, no time or frequency framming is then needed. Thus, either the transmision speed or the spectral efficiency will increase in the link. This is the case of the BPSK time reference beamforming faced in this section. The signal enhancer in figure (1) should consist then of a BPSK demodulator-modulator pair in order to regenerate the noisy received data.

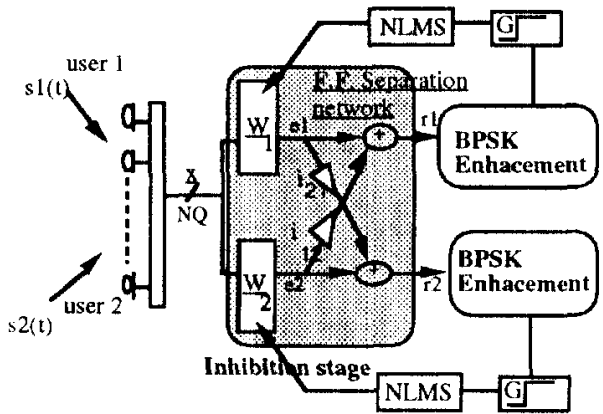

Figure 2.Proposed two-stage architecture for BPSK TRMB

In order to allow simultaneous user services, there should be enough SNIR at each branch of each BPSK enhancer to catch a different user. For this purpose the two-stage architecture of figure (2) is proposed. Because of the delays inherent in a FB network, the FF network is the more suitable one.to allow RF digital references separation.

In a BPSK communication link it is known that bellow $11 \mathrm{dBs}$ of EbNo, the error probability is not low enough to garantee a good quality transmission $\left(\mathrm{p}_{\varepsilon}>=10^{-}\right.$ 5)). The beamformer gain helps to lessen the SNR requirements (no more than $S N R=5 \mathrm{~dB}$ in an array of 10 sensors is necessary for $\mathrm{p}_{\varepsilon}=10^{-5}$ ). Thus, if a TRMB system is designed, each TRB should mantain this commented performance. Before presenting the obtained results. The utilized FF separation network is first described for the noiseless situation.

3.1. FF separation network

The input to the FF network can be equated as follows (2):

$$
\begin{aligned}
& \mathrm{e}_{1}=\mathrm{w}_{1}^{\mathrm{H}} \cdot \mathrm{x} \\
& \mathrm{e}_{2}=\mathrm{w}_{2}^{\mathrm{H}} \cdot \mathrm{x} \text { (2) e=A.x (3) }
\end{aligned}
$$

which, by substituting the complex data $x$ (1), reduces to the linear complex mixture in (3). From the inputs e, the separating network implements (4):

$$
\mathrm{r}_{1}=\mathrm{e}_{1}+\mathrm{i}_{12 \cdot \mathrm{e}_{2}}
$$$$
r_{2}=i_{21} \cdot e_{1}+e_{2} \quad \text { (4) } \quad r_{i}=s_{j} ; \quad i, j=1,2 \quad(5)
$$

aiming to obtain those inhibition weights $i_{i j}$ which separate the impinging references $\mathrm{s}_{\mathbf{i}}(5)$.

Several are the authors who have faced this source separation problem [7]. We are mainly interested in a low computational solution. Therefore we have resorted to recursive algorithms. Moreover, as the FF network will be cascaded with an adaptive beamformer, the recursive algorithm should be of low complexity regarding its time constants and other required adjustments.

One possibility is the one reported in [8] for the two source case. The obtained solution for the weights had the form in (6):

$$
i_{i j}=\frac{E\left\{\left|r_{i}\right| r_{i_{i}} *_{j}\right\}}{E\left\{\left|r_{i}\right| r_{j} \mid r_{j}\right\}} \quad\left|i_{i j}\right|<1
$$

which was then computed recursively by the same rule as the one proposed by [2].

In a further refinement of the problem, the authors found that the above rule is inadequate since it does not force the nulling of the cross-forth order cummulant. In order to match the rule in (6) with the objective of (7), equation (8) has to be fulfilled:
$\operatorname{cum}\left\{\left.\mathbf{r}_{1} \cdot \mathbf{r}_{2} \cdot \mathrm{I}_{2}\right|^{2}\right\}=0$
(7)
$E\left\{r_{1} \cdot r_{2}{ }^{*}\right\}=0$

Therefore, if one of the weights (9) is computed to fulfil (8):

$$
\begin{aligned}
& i_{12}=\frac{-E\left\{e_{1} \cdot e_{2}^{*}\right)+i_{21} \cdot E\left\{e_{1} \cdot e_{1}^{*}\right\}}{E\left\{e_{2} \cdot e_{2}{ }^{*}\right\}+i_{21}{ }^{*} \cdot E\left\{e_{2} \cdot e_{1}^{*}\right\}} \\
& i_{21}^{n+1}=i_{21}^{n}+\mu \frac{\left|r_{2}\right|^{2} \cdot r_{2}^{*} \cdot r_{1}}{E\left[\left.\left|r_{2}\right|^{2} \cdot r_{2}\right|^{2}\right\}}
\end{aligned}
$$

and the other (10) to fulfil (7), the separation will be always achieved (figure 6) even though high sensibilitydue to low coupling among references $\mathbf{s}_{\mathbf{i}}$ - is required.

\subsection{Bpsk TRMR. Simulations.}

In the TRMB system depicted in figure (2), the separating network is integrated with the adaptive beamforming (NLMS). As commented before, the goal is to obtain a sufficient SNIR at each BPSK enhancer input to avoid collision among demanding users. The main problem arises from the general bad behaviour of the separating networks in front of a noisy input (increase of the variance and bias in the inhibition weights [1]).

- For instance, in the scenario where simulation of figure (7) was performed, a SNR of $5 \mathrm{dBs}$ for each impinging signal was enough for the adaptive beamforming (NLMS) (A line) to reinforce the poor SIR, that was first obtained 
at each branch by the inhibitory weights. Then, the BPSK enhancer (optimization stage) was connected (B line). Each enhancer cleaned then each reference from the noise and allowed the beamformer to increase sufficiently the SNR (up to $11 \mathrm{~dB}$ ) to obtain a $p_{\varepsilon}$ less than $10^{-4}$ for each user.

Nevertheless, in other simulations, the performance was not always so good. It highly depended on an adequate signal power, SNR and coupling in the initial mixture,A. More robust results will be obtained if a frequency fraiming TRB is used, which will solve better the noise enhacement, even in a BPSK link.

\section{FREQUENCY FRAMING TRB}

In a frequency framing context, the kind of temporal reference selected by the system is a frequency carrier. Whether this carrier must be modulated or not is left open to the communication link specifications. Quite frequently, the communication system may dedicate a specific channel where users issue their unmodulated carrier reference only for user location purposes (calling tone). On the other hand, if it is a BPSK link, another possibility is to extract the modulated carrier by squaring and filtering the received signal. Thus, frequency framing TRB is rather versatile. In the following, it will be put forward an sepecific frequency framing two-stage architecture in order to allow simultaneous TRB. When compared with the BPSK Time Reference Multibeamformer, the reader will appreciate its lower algorithmic complexity together with its higher robustness and better performance.

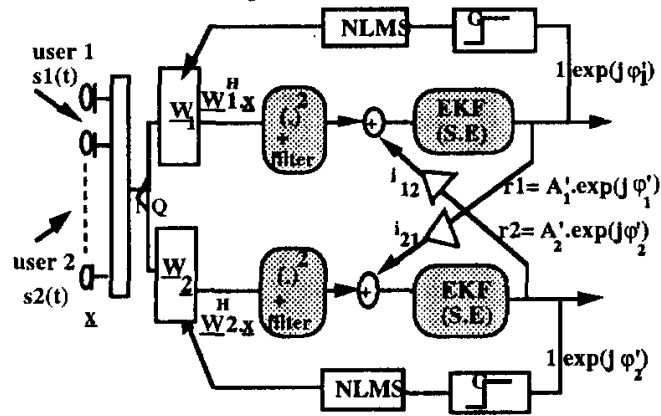

Figure 3. Frequency framming TRMB for BPSK signals

For the local regeneration stage, normally an analog PLL is used for the purposes of unmodulated carrier regeneration. Because the system we are dealing with has to be able to track amplitude, phase and frequency an specific Extended Kalman Filter (EKF) will be used [9] for the case of complex (i-q components) sinusoids. The EKF not only estimates the incoming signal sample but also predicts the next one. This feature together with its robustness and good performance (able to acquiere and track carriers with SNR of $-5 \mathrm{~dB}$ in a bandwith of $4 \mathrm{Khz}$ [9]) is considered by the authors as crucial for a successful performance of the proposed simultaneous TRMB depicted in figure (3). The reader will note two main differences with respect to the scheme in figure (2). On the one hand, as the EKF predicts the next signal sample, it may compensate the inherent delay of the used FB separating network, thus this structure can be used instead of the FF one in order to speed up the convergence. On the other hand, the EKF regenerates the reference not only in phase but also in amplitude. Hence, it delivers a reference to both, the adaptive algorithm and the separating FB network. This last aspect will be of key importance for a good source separation performance in the realistic noisy case.

\subsection{Separating FB network}

For a complete design of the inhibition weights in the FB network we refer to [6]. The authors resort to the uncorrelated nature of the issued user reference estimates, $r_{i}$. Thus, the criterion is (11):

$$
\phi=\left|\mathrm{E}\left\{\mathrm{r}_{\mathrm{k}} \cdot \mathrm{r}_{\mathrm{j}}^{*}\right\}\right|_{\min }^{2} \quad \mathrm{k} \neq \mathbf{j} \quad \mathrm{k}, \mathrm{j}=\mathbf{l} \text {...NS }
$$

which, applied to the NS users, leads to the following adaptation rule for the weights in (12):

$$
i_{k j}^{n+1}=i_{k j}^{n}+\mu \cdot \frac{\mid r_{j} j^{2} \cdot r_{j} \cdot r_{k}}{E\left\{\left.\left|r_{j}\right|^{2} \cdot I_{k}\right|^{2}\right\}}
$$

\subsection{Frequency framing TRMB. Simulations}

In the TRMB (figure 3), in order for all the users to be served, the separating network will introduce the necessary SIR at the input of each EKF. That is, statistical independence is used to make the system start; each enhancer will catch up a different reference because of the assymety introduced by the inhibitory weights. To illustrate the robust performance of the system a filled linear array of 10 sensors in a very low SNR scenario has been chosen: the two sent references have a normalised frequency of 0.1 and 0.15 and impinge from an elevation angle of $0^{\circ}$ and $8^{\circ}$ with $S N R$ of $-5 \mathrm{~dB}$ and $-3 \mathrm{~dB}$ respectively.

- Figure (4) shows each beamformer evolution; the A-line plots the beamformer after 20 snapshots, the B-line corresponds after 1000 snapshots. Note that convergence is slow and nulling slight due to the low SNR.

- Figure (5) plots the estimated reference frequencies compared to the actual one in dotted line. Note that the multiuser system is helped by the beamformer; the reason can be found in the SNIR increment that the beamformer immediately introduces (20 snaphots). On the other band, from the authors experience, in this scenario the EKF and NLMS combination never loses the desired signal in the global scheme. This contrasts with the case of having only two sensors, which fails quite frequently at such low SNR. In any case, if the signal enhancer regenerates the desired reference not from the output of a single sensor but from the output of the array $\left(\mathbf{w}_{i}^{\mathbf{H}} \cdot \mathbf{x}\right)$, the system will be always protected from an specific sensor misfunction. 


\section{CONCLUSIONS}

In many real situations, the well-known source separation problem has to be solved in noisy scenarios. For this reason, this work sets out to introduce a second enhancing stage, claimed by the Kolmogorov's theorem. Specifically, for simultaneous multiuser identification, the frequency framing TRB turns out to be the more successfull system, even for BPSK signals. Its robust performance is owed to the full integration that exits among: the separating network, the spatial descrimination (beamformer)and the digital PLL

Figure 4. Frequency framing TRMB. Array: 10 sensors; Signals: signal $1->$ at $2^{\circ}(S N R=-5 d B$, normalize freq. $=0.1)$; signal 2-> at $8^{\circ}(S N R=-3 d B$, normalize freq. $=0.15$ ).

A: Beamformer afier 20 snapshors.

B:Beamformer after 1000 snapshots
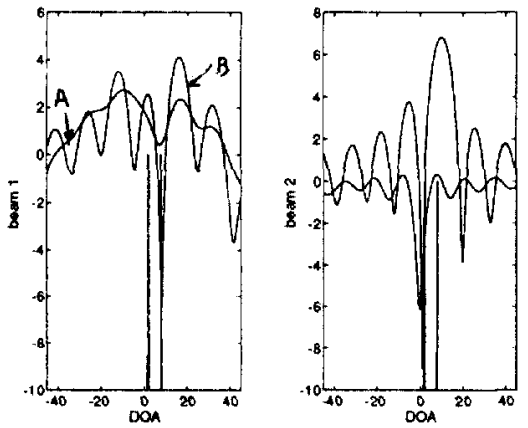

Figure 5. Frequency framing TRMB.The same simmulation as in figure 5 , frequency estimation
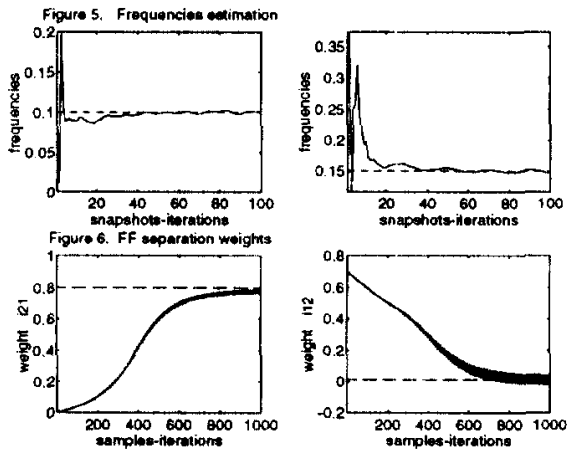

Figure 6. FF separation network in a noiseless case Computation with equeations (9) and 110 both recursive .Two tones both of amplitude 1. Mixing matrix

$A=\left[\begin{array}{cc}1 & -0.01 \\ 0.8 & 1\end{array}\right] ;$ estimated values of the inverse $=[0.013,0.78]$
Figure 7. BPSK TRMB. Array: 10 sensorsisignals: signal $1 \rightarrow$ at $7^{\circ}(S N R=5$ dB, normalize freq. $=0.05)$; signal 2-> at $17^{\circ}(S N R=5$ dB, normalize freq. $=0.05)$.

A: Beamformer after trainning with the separatine_network. $B:$ Beamformer after connecting the BESK enhancer(noise removal).
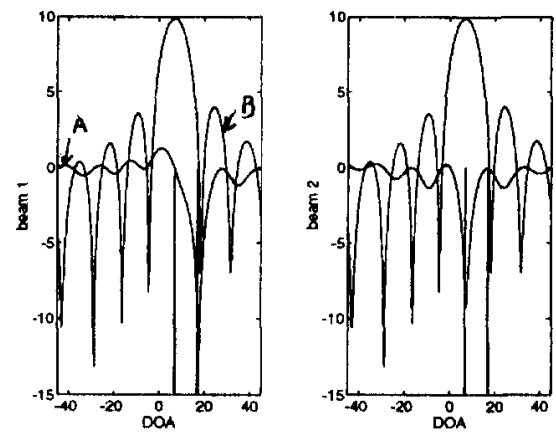

\section{REFERENCES}

[1] Bar-Ness Y. , "Bootstrapping Adaptive Interference Cancelers: Some Practical Limitations," Proc. Globcom'82, pp, 1251-1255.

[2] C.Jutten,J.Herault,P.Comon," Blind separation of sources, Parts I,II,"Signal Processing 24,pp.120,Elsevier 1991

[3] M.Feder,E.Weinstein,"Parameter estimation of superimposed signals using the EM algorithm,"IEEE Trans. ASSP, vol.ASSP-36,pp.477-489,1988.

[4] A.N.Kolmogorov,"On the representation of continuous functions of many variables by superposition of continuous functions of one variable and addition," (in Russian), Dokl.Akad.Nauk.,USSR,114,pp.953$956,1957$.

[5] Nájar M. Lagunas M. A. , Pérez-Neira A. , "Source Separation based on coupled single DOA Estimation Processors", Proc. ICASSP-93, pp. IV336-IV339, 1993.

[6] Lagunas M. A. .Pagés A. , "Multitone tracking with coupled EKFs and high order learning". Proc. ICASSP92, pp. V153-V156,1992.

[7] P.Comon,J.Lacoume,"Statistiques d'ordres supèrieurs pour le traitement du signal," Cours dans "Traitement du Signal-Developpements Recents," Faciucule 3/4,pp. 4256. Les Houches 1993.

[8] Ana Pérez-Neira,M.A.Lagunas, "HOS in Antenna Array Applications: TRMB," ATHOS Workshop on:"System Identification and HOS, Sophia Antipolis (France) 1993.

[9] J.Fernandez, M.A.Lagunas,G.Vazquez,"Breadboarding of a digital beamforming network," ERA-ESA ESTEC Technical repor, European Spatial Agency, Doc $\# 1987 / 01,1990$. 